\title{
Malignancy Rate and Malignancy Risk Assessment in Different Lesions of Uncertain Malignant Potential in the Breast (B3 Lesions): An Analysis of 192 Cases from a Single Institution
}

\author{
Svjetlana Mohrmann ${ }^{a} \quad$ Anna Maier-Bode $^{\mathrm{a}}$ Frederic Dietzel $^{\mathrm{b}}$ Petra Reinecke $^{\mathrm{c}}$ \\ Natalia Krawczyk ${ }^{a}$ Thomas Kaleta ${ }^{a}$ Ulrike Kreimer $^{\mathrm{a}} \quad$ Gerald Antoch $^{\mathrm{b}}$ \\ Tanja N. Fehm ${ }^{a}$ Katrin Sabine Roth ${ }^{\text {b, d }}$ \\ ${ }^{a}$ Department of Gynecology and Obstetrics, Medical Faculty, Interdisciplinary Breast Centre, University of

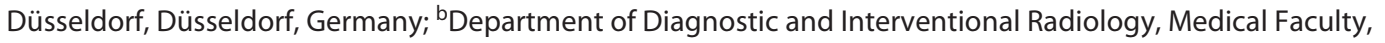 \\ University of Düsseldorf, Düsseldorf, Germany; ' Institute of Pathology, Medical Faculty, University of Düsseldorf,

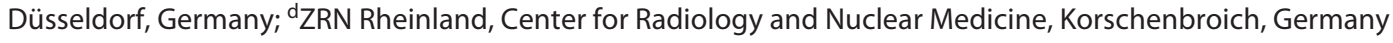

\section{Keywords}

B3 lesion · Malignancy rate $\cdot$ Risk factors · Breast surgery $\cdot$ Therapy

\footnotetext{
Abstract

Background: The question of how to deal with B3 lesions is of emerging interest. Methods: In the breast diagnostics of 192 patients between 2009 and 2016, a minimally invasive biopsy revealed a B3 lesion with subsequent resection. This study investigates the malignancy rate of different B3 subgroups and the risk factors that play a role in obtaining a malignant finding. Results: The distribution of B3 lesions after minimally invasive biopsy was as follows: atypical ductal hyperplasia $(\mathrm{ADH}), 7.3 \%$; flat epithelial atypia (FEA), 7.8\%; lobular neoplasia (LN), 7.8\%; papilloma ( $\mathrm{Pa}), 49.5 \%$; phylloidal tumour (PT), 8.9\%; radial sclerosing scar (RS), 3.1\%; mixed findings, 10.4\%; and other B3 lesions, 5.2\%. Most B3 lesions were detected by stereotactic vacuum-assisted biopsy (44.3\%), 36.5\% by ultrasound-assisted biopsy, and $19.3 \%$ by magnetic resonance imaging-assisted biopsy. Most B3 lesions (55.2\%) were verified by surgical resection, whereas $30.7 \%$ were downgraded to a benign lesion. About $14.1 \%$ of the cases were upgraded to malignant lesions, $9.4 \%$ to ductal carcinoma in situ and $4.7 \%$ to invasive carcinoma. In relation to individual $\mathrm{B} 3$ lesions, the following malignancy rates were found: $28.6 \%(A D H), 13.3 \%$ (FEA), 33.3\% (LN), $12.6 \%$
}

(Pa), 5.9\% (PT), and 0\% (RS). The most important risk factor was increasing age. Postmenopausal status was considered an increased risk for an upgrade $(p=0.015)$. A known malignancy in the ipsilateral breast was a significant risk factor for a malignant upgrade ( $p=0.003$ ). Conclusion: Increasing knowledge about B3 lesions allows us to develop a "lesionspecific" therapy approach in the heterogeneous group of B3 lesions, with follow-up imaging for some lesions with less malignant potential and concordance with imaging or further surgical resection in cases of disconcordance with imaging or higher malignant potential.

(C) 2021 The Author(s) Published by S. Karger AG, Basel

\section{Introduction}

B3 breast lesions represent only a very small group of breast diseases but have a special position concerning their malignant potential. Non-operative core needle biopsy was introduced in the mid-1990 to assess uncertain breast lesions in imaging trying to give a definitive histological diagnosis and recommend a further therapy/open resection, if necessary [1]. The B-classification system for evaluating core needle biopsies of the breast was established in 1999 by 23 European pathologists to achieve consistency in diagnosing breast diseases and define the prognostic features of carcinomas [2]. The system briefly mercial purposes requires written permission. 
comprises five categories: B1, normal tissue or unsatisfactory; $\mathrm{B} 2$, representative and benign lesion; B3, benign lesion with unknown biological potential; B4, suspicious for malignancy; and B5, malignant. The heterogeneous group of B3 lesions includes the following different histopathological entities: atypical ductal hyperplasia (ADH; also known as atypical intraductal proliferation), flat epithelial atypia (FEA), lobular neoplasia (LN), papilloma $(\mathrm{Pa})$, phyllodes tumour (PT), radial sclerosing scar (RS) and others, based on the World Health Organization (WHO) classification (fourth edition, 2012). In the new WHO classification of breast tumours (fifth edition, 2019), LN is divided into atypical lobular hyperplasia (ALH) and lobular carcinoma in situ (LCIS) based on the widening and filling of the terminal ducto-lobular unit. After complete surgical resection of a B3 lesion, the overall risk for malignant upgrade is $9.9-35.1 \%$ in various studies [3-6]. Due to their unclear biological potential, heterogeneous histological features correspond to different malignancy potential, and there is a lack of consensus on how this finding should be further clarified or closely observed. Therefore, the decision on how to proceed with a B3 lesion is highly complex. There are numerous, mostly retrospective, studies that deal with $\mathrm{B} 3$ lesions, presenting data from open surgical excisions. The latest studies show a trend towards extended vacuum biopsy as therapy, the restraint of open surgery and subsequent appropriate monitoring [7-9]. At the Second International Consensus Conference on lesions of uncertain malignant potential in the breast, it was decided that follow-up after a B3 lesion should be based on the patient's risk for an upgrade of the $\mathrm{B} 3$ lesion after further diagnosis. With an underestimation rate of $5 \%$ for invasive carcinoma or more than $10 \%$ for ductal carcinoma in situ (DCIS), it was decided that "wait and see" is not an appropriate therapy regimen. Instead, another biopsy in the form of a vacuum biopsy or an open surgical excision should be performed [10].

To enhance the knowledge about B3 lesions and present data for further improvement of therapy regimen, this study investigated the malignancy rate of 192 B3 lesions from our breast centre and tried to identify risk factors that indicate an upgrade to malignancy.

\section{Materials and Methods}

In this retrospective study, data from daily records of patients treated between January 2009 and December 2016, with a diagnosis of B3 lesions, treated in our academic interdisciplinary gynecoradiological unit were collected and analysed. The inclusion criterion was a histologically diagnosed B3 lesion followed by surgical resection of the surrounding tissue. The indication for surgical resection of the B3 lesion was performed in the clinicopathological conference, where concordance of imaging and histology was discussed. The exclusion criteria were missing surgical resection
Table 1. Data of patients that received biopsies characterised as B3 lesion $(n=192)$

\begin{tabular}{|c|c|}
\hline Patient age, years, range (mean; SD) & $13-83(52 ; 13.83)$ \\
\hline \multicolumn{2}{|l|}{ Sex } \\
\hline Female & $189(98.4 \%)$ \\
\hline Male & $3(1.6 \%)$ \\
\hline \multicolumn{2}{|l|}{ Endocrine status* } \\
\hline Premenopausal & $90(47.6 \%)$ \\
\hline Postmenopausal & $99(52.4 \%)$ \\
\hline \multicolumn{2}{|l|}{ Known breast malignancy } \\
\hline None $^{+}$ & $143(74.5 \%)$ \\
\hline Invasive breast cancer & $41(21.3 \%)$ \\
\hline DCIS & $8(4.2 \%)$ \\
\hline \multicolumn{2}{|l|}{ High-risk breast cancer group } \\
\hline Not fulfilling criteria & $148(77.1 \%)$ \\
\hline Fulfilling criteria-high-risk group & $44(22.9 \%)$ \\
\hline \multicolumn{2}{|l|}{ Side of breast } \\
\hline Left & $100(52.1 \%)$ \\
\hline Right & $92(47.9 \%)$ \\
\hline \multicolumn{2}{|l|}{ Location of lesion in breast } \\
\hline Upper outer quadrant & $74(38.5 \%)$ \\
\hline Lower outer quadrant & $39(20.3 \%)$ \\
\hline Lower inner quadrant & $25(13 \%)$ \\
\hline Upper inner quadrant & $31(16.1 \%)$ \\
\hline Central/retromamillar & $16(8.3 \%)$ \\
\hline More than one quadrant & $7(3.6 \%)$ \\
\hline \multicolumn{2}{|l|}{ Invasive diagnostic } \\
\hline Stereotactic-assisted vacuum biopsy & $85(44.3 \%)$ \\
\hline $\begin{array}{l}\text { Ultrasound-assisted high-speed core } \\
\text { needle biopsy }\end{array}$ & $70(36.5 \%)$ \\
\hline MR-assisted vacuum biopsy & $37(19.3 \%)$ \\
\hline \multicolumn{2}{|l|}{ Microcalcifications in specimen } \\
\hline Yes & $114(59.3 \%)$ \\
\hline No & $83(40.6 \%)$ \\
\hline
\end{tabular}

${ }^{*}$ Men were excluded from this analysis; ${ }^{+}$no malignant cells.

(mostly in cases with concordance between imaging with histological finding in the clinicopathological conference) or surgery in a different hospital. Bilateral B3 lesions were counted as two different lesions. The local ethics committee approved this retrospective analysis.

\section{Data Analysis}

The analysed data comprised age, sex, premenopausal or postmenopausal, positive family anamnesis for breast cancer, participant of high-risk breast cancer screening, prior malignancy of the breast, lesion side, location of the lesion in the breast, palpable lesion, microcalcifications, and breast secretion (Table 1).

\section{Clinical Examination, Imaging, and Biopsy}

Past medical history and clinical breast examination was taken and performed prior to imaging of each patient. Breast ultrasound, two plain or digital full-field mammography and magnetic resonance (MR)-mammography were performed and evaluated by 3 gynecologists with 5-12 years of experience and/or breast radiologists with 6 and 10 years of experience.

After local anaesthesia, biopsy of the suspicious breast lesion was performed under ultrasonographic, mammographic, or MRmammographic guidance, and marked with a clip, after biopsy. 
Detailed information regarding these procedures is given in the supplemental material (for all suppl. material, see www.karger. com/doi/10.1159/000517109.

\section{Histopathological and Statistical Analyses}

Two board-certified pathologists specialised in breast pathology with more than 5 years of experience performed histopathological analysis on hematoxylin-and-eosin-stained sections or additional immunohistochemically stained sections with CK 5/6, CK 8/18, p63, and E-cadherin. The histopathological diagnosis was obtained from the original pathology report. Reports were based on the fourth edition of the WHO classification.

SPSS version 24.0 (IBM, Armonk, NY, USA) was used for statistical analysis. Descriptive statistics were reported. KolmogorovSmirnov and Shapiro-Wilks tests were used to determine normal distribution. Pearson's $\chi^{2}$ test and binary logistic regression were used to detect significant risk factors for a malignant upgrade.

\section{Results}

Breast biopsies $(n=192)$ of 175 women and 3 men were included in the analysis. Fifteen women $(7.8 \%)$ received two biopsies; bilateral biopsy was performed in 8 of them (4.2\%). Three suspicious lesions were clarified with a breast biopsy in 1 woman (0.6\%). Each biopsy was counted individually. Patients' age ranged from 13 to 83 years (mean, 52 years; standard deviation, 13.83). A total of 189 biopsies diagnosed with a B3 lesion were taken from female patients; 3 men were diagnosed with a B3 lesion in their histopathological analysis. Ninety (47.6\%) women with diagnosed B3 lesions were premenopausal, and 99 (52.4\%) women had a postmenopausal status. Male patients were excluded in this subanalysis. Fortynine lesions were detected in patients already diagnosed with invasive breast cancer $(n=41)$ or DCIS $(n=8) ; 159$ patients with B3 lesions had no prior breast malignancy. In 14 patients $(7.3 \%)$ with $\mathrm{B} 3$ lesion, the malignancy was located in the ipsilateral breast; in 33 patients $(17.2 \%)$ with B3 lesion, the malignancy was located in the contralateral breast, and 2 patients (1\%) had bilateral malignancies. Forty-four patients (22.9\%) with diagnosed B3 lesions fulfilled the criteria for inclusion in the high-risk breast cancer group according to the German Consortium requirements for Hereditary Breast and Ovarian Cancer. The anamnesis of 148 patients with diagnosed B3 lesions was not suspicious of genetic cancer risk for hereditary breast and ovarian cancers.

B3 lesions were found in 100 breast biopsies (52.1\%) in the left breast, and 92 B3 lesions (47.9\%) were detected in the right breast. Most B3 lesions were detected in the upper outer quadrant (74 cases; 38.5\%), 39 B3 lesions (20.3\%) were located in the lower outer quadrant, 25 B3 lesions (13\%) were found in the lower inner quadrant, and $31 \mathrm{~B} 3$ lesions (16.1\%) were detected in the upper inner quadrant. Sixteen B3 lesions (8.3\%) were found in the central/retromamillar region, and $7 \mathrm{~B} 3$ lesions (3.6\%) were located in more than one quadrant.

Most B3 lesions were detected with stereotactic vacuum-assisted biopsy (85 cases; 44.3\%), 70 B3 lesions $(36.5 \%)$ were diagnosed with an ultrasound-assisted high-speed core needle biopsy, and 37 B3 lesions (19.3\%) were detected with MR-assisted vacuum biopsy.

\section{Histopathology of B3 Lesions}

Among B3 lesions, $\mathrm{Pa}$ was the most frequent lesion with 95 cases $(49.5 \%)$. Pa of the breast are characterised as proliferative fibrovascular tissue branches with an overlying layer of epithelial and myoepithelial cells. PTs, biphasic tumours with a leaf-like architecture, hypercellular stroma, and clef-like epithelial covered spaces, were found in 17 biopsies (8.9\%). FEA, histopathologically identified by an atypical epithelial proliferation of epithelial cells without architectural atypia, and classical LN, comprising a wide variety of atypical epithelial proliferation originating from the acinar structures of the breast, were detected in 15 biopsies (7.8\%). Variants of LN (nonclassical LCIS) were excluded from the analysis, as they represent a B5 lesion. Fourteen biopsies (7.3\%) were diagnosed as $\mathrm{ADH}$, characterised by intraductal clonal proliferation of epithelial cells. Entrapped ducts neighboured by radiating ducts and lobules indicate RS detected in 6 biopsies (3.1\%). An overview of the histopathology of the most frequent lesions is displayed in Figure 1. Twenty biopsies $(10.4 \%)$ were diagnosed as mixed lesions containing $\mathrm{ADH}$ and $\mathrm{FEA}$ in 3 cases (1.6\%), $\mathrm{ADH}$ and $\mathrm{Pa}$ in 6 cases (3.2\%), $\mathrm{LN}$ and $\mathrm{Pa}$ in 1 case (0.5\%), and $\mathrm{RS}$ and $\mathrm{Pa}$ in 8 cases (4.2\%). As "others," 10 biopsies (5.2\%) were summarised as 3 cases (1.6\%) with fibroepithelial tumours and 1 biopsy (0.5\%) classified as epithelial proliferation, 1 with tubular proliferation, 1 with myofibroblastic tumour, 1 with adenomyoepithelioma, 1 granular cell tumour with malignant cells, and 1 pregnancy-like hyperplasia with atypical cells. Microcalcifications were detected in the tissue of 114 biopsies (59.3\%). In 78.6\% $(n=11)$ of diagnosed ADH and $93.3 \%(n=14)$ of FEA, microcalcifications were detected. In contrast, only in 2 cases of PT (11.8\%) were non-suspicious microcalcifications found on mammograms.

\section{Upgrade/Downgrade after Surgery}

Most B3 lesions (106 cases; 55.2\%) were verified with surgical resection, whereas 59 B3 lesions (30.7\%) were downgraded to a benign B2 lesion. No further suspicious tissue was found after surgical resection, so it was assumed that the B3 lesion was totally resected via biopsy. After surgery, 18 B3 lesions (9.4\%) were upgraded to DCIS, and 9 B3 lesions (4.7\%) turned out to be co-located with invasive breast cancer. 


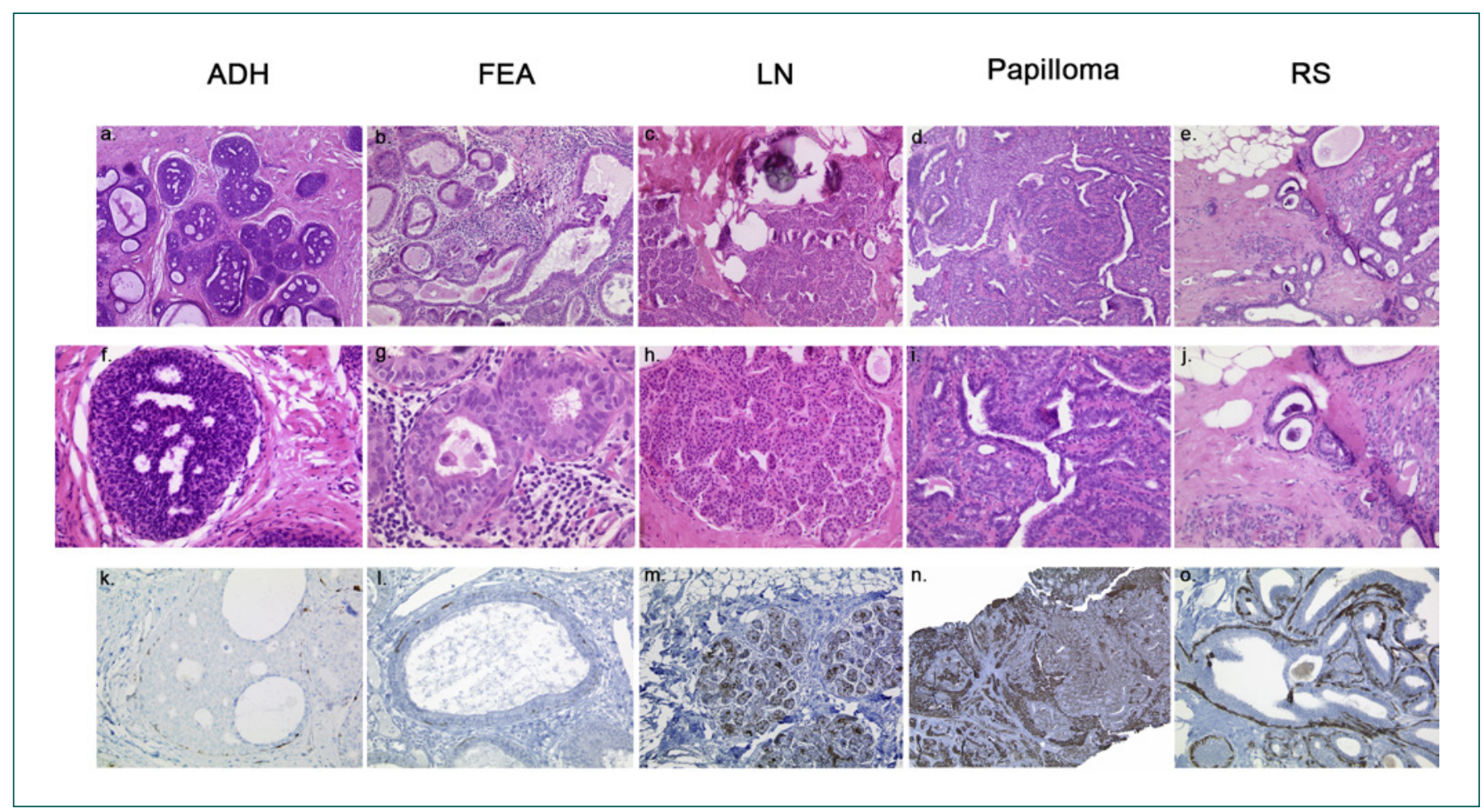

Fig. 1. a, $\mathbf{f} \mathrm{HE}$ staining of $\mathrm{ADH}$ shows intraductal epithelial clonal cell proliferation smaller or equal to $2 \mathrm{~mm}$ size. $\mathbf{b}, \mathbf{g}$ HE-stained specimen with the diagnosis of FEA characterised by one or several layers of disorganised atypical epithelial cells, which are cuboidal or columnar, but with normal architecture. c, $\mathbf{h}$ Intracytoplasmic mucin vaculola in $\mathrm{HE}$ staining of $\mathrm{LN}$, with characteristic lobulocentric proliferative small and monomorphic cells filling the expanded acini of the affected lobule. $\mathbf{d}$, $\mathbf{i}$ HE staining of papilloma displaying proliferation of fibrovascular core with layers of myoepithelial and epithelial cells. e, $\mathbf{j}$ Radial scar stained with HE characterised by fibroelastic core, with entrapped and radiating ducts. k-o Immunohistochemical staing with cytokeratin 5 shows myoepithelial and luminal epithelial cells in normal breast tissue. This staining helps to differentiate B3 lesions from DCIS and infiltration ductal carcinoma.

\section{LN as a Significant Risk Factor for an Upgrade}

In total, $27 \mathrm{~B} 3$ lesions (14.1\%) were upgraded to a malignant lesion. Four cases $(28.6 \%)$ characterised as ADH after biopsy were upgraded to a malignant lesion (3 cases with DCIS and 1 case with invasive ductal carcinoma IDC). Two biopsies (13.3\%) diagnosed as FEA were upgraded to DCIS and IDC. Five cases (33.3\%) described as LN after biopsy were upgraded to a malignant lesion: 3 cases with DCIS, 1 IDC, and 1 invasive lobular carcinoma (ILC). Twelve cases (12.6\%) formerly characterised as $\mathrm{Pa}$ had to be upgraded, consisting of 9 DCIS cases and 3 IDC. One PT (5.9\%) turned out to be an ILC after surgery. None of the biopsies diagnosed as RS had to be upgraded. A significant correlation was found between the detection of LN as B3 lesion in biopsy and an upgrade to a breast malignancy ( $p=0.039$; suppl. Fig. 1 ). Patients with LN have a 3.2-fold higher risk for and upgrade to a malignant breast lesion after surgery than patients with other B3 lesions.

Binary logistic regression analysis was performed without mixed lesions and "other" B3 lesions, and the group of $\mathrm{Pa}$ was taken as the reference group. Age turned out to be a significant risk factor for upgrading a B3 lesion to a breast malignancy $(p=0.001)$. All B3 lesions $(n=2 ; 100 \%)$ in patients aged $>80$ years and $25.5 \%(n=$ 13) of B3 lesions in patients aged between 61 and 80 years were upgraded to a breast malignancy. Ten B3 lesions (9.6\%) in patients aged between 41 and 60 years and 2 B3 lesions $(6.7 \%)$ in patients aged $21-40$ years were malignant. No malignancy was detected in patients aged $<20$ years.

Postmenopausal status was another significant risk factor for a malignant upgrade of a B3 lesion $(p=0.015)$. In premenopausal patients, a malignant lesion was detected in $7.8 \%(n=7)$. In 20 postmenopausal women (20.2\%), B3 lesions were upgraded to malignancy.

Binary logistic regression analysis showed no significant risk for an upgrade into a malignancy if microcalcifications were present in the biopsy ( $p=0.18$; odds ratio $=2.12$ ) or in patients with known breast malignancy. In $42.9 \%$ of the patients $(n=6)$ with a known malignancy in the ipsilateral breast, a B3 lesion in biopsy was upgraded to a malignant lesion, with the same histology as the primary tumour. In comparison, in 2 B3 lesions (6.1\%) 
diagnosed in patients with contralateral breast cancer and 1 patient $(50 \%)$ with bilateral breast cancer, a malignant upgrade was performed after surgery. A known malignancy in the ipsilateral breast turned out to be a significant risk factor for a malignant upgrade of a B3 lesion $(p=0.003)$.

\section{Discussion}

The management of the heterogeneous group of B3 lesions in the breast is still under debate due to the lack of knowledge of their malignant potential and risk factors. In this study, 192 B3 lesions of patients were analysed to determine the potential risk factors of a malignant upgrade of each B3 lesion with regard to the development of a "lesion-based" therapy.

Twenty-seven of 192 (14.1\%) of the cases histologically classified as B3 preoperatively proved malignant in the final histological analysis. Thus, the results agree with the previously published data $[4,5,11]$.

The most frequent lesion in the punch biopsy was $\mathrm{Pa}$, with 95 cases (49.5\%). After surgical excision, 12 cases were upgraded with an upgrade rate of $12.6 \%$ in our study. The evaluation of 34 studies in a meta-analysis showed an upgrade rate for $\mathrm{Pa}$ of $7 \%$ compared to $36.9 \%$ for atypical $\mathrm{Pa}$; studies after 2005 even showed a lower tendency to upgrade [12], similar to this study. Atypical $\mathrm{Pa}$ are also known as $\mathrm{Pa}$ with atypia including $\mathrm{ADH}$ and/ or DCIS. They are characterised by few or no myoepithelial cells and a focal population of monotonous cells with cytological and architectural features of low grade ductal neoplasia. $\mathrm{ADH}$ is diagnosed if the area of atypia is less than $3 \mathrm{~mm}$, otherwise the diagnosis is DCIS. Therefore, radiological monitoring seems to be acceptable for smaller or completely excised benign intraductal $\mathrm{Pa}$ [13] or, as an alternative to open surgical excision, complete/extended vacuum excision [14].

PT is the second most common lesion detected by ultrasound-assisted high-speed core needle biopsy in this study, with 17 cases (8.9\%) and 1 ILC after surgical excision (upgrade rate of 5.9\%). In our study, PT was found more frequently than reported in the literature $(1-2 \%$ of all breast biopsies) [10], but with a similar upgrade rate as previously published [15]. Clinically, a PT is most often conspicuous by a palpable, fast-growing tumour that is usually surgically removed. Regardless of the subtype of a phylloidal tumour in the histological analysis, surgical extirpation or even more radical surgery in the form of a mastectomy must be performed [16].

In the literature, heterogeneous data concerning FEA exist regarding incidence and malignancy rate. This is partly because FEA in studies only with $\mathrm{ADH}$ is summarised as ductal intraepithelial neoplasia or atypical in- traductal epithelial proliferation, which in addition to $\mathrm{ADH}$ and FEA includes columnar cell hyperplasia with atypia [17]. FEA was found in 15 cases and thus in $7.8 \%$ of B3 lesions. Older studies showed significantly higher rates of $18.3-34.4 \%[6,18]$, whereas newer studies that consider the pathological-histological correlation showed rates of less than $10 \%[19,20]$. Surgical excision is unnecessary if the microcalcifications are removed completely [13] while using $9 \mathrm{G}$ or $11 \mathrm{G}$ vacuum biopsy. Because our breast centre had an upgrade rate of $13.3 \%$, the procedure performed was considered appropriate.

This study treated LN in the same way as Menon et al. [21] who considered LCIS and ALH together as classical LN, with the two entities differing only in the widening and filling of TDLU [22]. Although the new WHO classification of breast tumours (fifth edition, 2019) divides LN into ALH and LCIS, a recent inter-observer study from Switzerland showed the most reproducible results when comprising ALH and LCIS as lobular intraneoplasia or LN [23]. As described, the differentiation into these entities can be of great importance for patients in the future, as the malignant update rates of ALH are much lower than the malignant update rates of LCIS [24]. In our study, we found a classical LN in 15 biopsies (7.8\%). Other studies showed values of $4-25 \%[6,14$, 18 , with other high-risk lesions often present in addition to classic LN. At 33.4\%, LN in this study has a significantly higher risk $(p=0.039)$ for an upgrade than all other B3 lesions. With an LN in the biopsy, the risk for an upgrade was 3.2 times higher in this study than for patients with other B3 lesions. The tremendous variability of upgrade rates of other studies from 0 to $66 \%[4,6$, $10,18,25$ ] can probably be explained by partially small case numbers or separate evaluation of LN or together with simultaneously present high-risk lesions, such as $\mathrm{ADH}$ [26]. Lower malignancy rates of 3-8\% refer to larger case numbers [26] or concordance between imaging and pathology [27]. This study could not demonstrate an association between an unconformity and a higher risk for an upgrade. Although different strategies are followed after detecting LN in the biopsy [10], our approach with excision was considered correct, given such a high malignancy rate.

In contrast to other studies $[6,18], \mathrm{ADH}$ was not the most common B3 lesion in this study. The 14 cases (7.3\%) in biopsy deviated significantly from other studies, where $\mathrm{ADH}$ accounted for about $40 \%$ of all B3 lesions. The malignancy rate of $28.6 \%$ does not differ significantly from other studies with rates of $18-50.4 \%$ and calls for the recommendation for excision after biopsy to be reconsidered $[6,10,20,28]$. The fact that $\mathrm{ADH}$ is considered an indicator lesion and that a differential diagnosis of DCIS and $\mathrm{ADH}$ is made solely based on size further reinforces this strategy [29]. 
Only a few studies $[6,14,18]$ with small case numbers of RS with $0.9-26 \%$ exist. This correlates well with this study's findings. RS was present in 6 cases $(3.1 \%)$ after a biopsy. The introduction of digital mammography and the improvement in sonographic resolution have shown an increased RS prevalence since 2000 [28]. Although the malignancy rate was $0 \%$, open excision is still recommended due to the controversial management and small database.

This study tried to identify other risk factors that influence the upgrade of all B3 lesions and found a higher risk for a malignant tumour in older postmenopausal women and patients with ipsilateral breast cancer. The risk for a malignant finding increased significantly with age $(p=0.001)$. Already after age 51 , the risk of being upgraded tripled, similar to Yu et al. [20], but only for ADH and FEA. To the authors' knowledge, this is the first study to describe an increased risk for a malignant upgrade in postmenopausal women with ipsilateral breast lesions.

Mixed lesions not found in the percentage distribution of findings are seen as possible limitations of the results. Even a subdivision of a B3 lesion by subclassification can significantly influence the malignancy rate within a B3 lesion $[17,30]$.

The results of this retrospective study do not show significant differences between malignancy risk and the different B3 lesions, although LN is more often associated with an increased risk, probably due to small case numbers and the heterogeneity of the lesion. Another possible reason for such a high upgrade rate could have been a preselection of cases in the clinicopathological conference, since surgery was only recommended for cases with discrepance between imaging and histology. A further limitation is that the risk factors could only be evaluated in relation to all $\mathrm{B} 3$ lesions due to the small case numbers of the individual B3 subtypes in few studies published so far. Thus, general and not subgroup-specific statements can be made on how individual factors increase the upgrade risk for all $\mathrm{B} 3$ lesions.

\section{Conclusion}

The diagnosis of a $\mathrm{B} 3$ lesion in breast biopsy always carries the risk of overlooking a more significant change. Especially in those cases which show a disconcordance after clinicopathological conference, a strategy with subsequent surgical intervention is pursued with the aim of complete resection of the lesion and correlation with imaging. Since our knowledge about $\mathrm{B} 3$ lesions is increasing, it is reasonable to develop a "lesion-specific" therapy approach in the heterogeneous group of B3 lesions, with follow-up imaging for some lesions, as solitary $\mathrm{Pa}$ and RS, or further surgical resection in cases of disconcordance or higher malignant potential, as also proposed in the new guidelines [31].

\section{Statement of Ethics}

The Ethics Committee of the University of Dusseldorf (Ref. No. 5019) approved the study. Individual patient informed consent was unnecessary as these anonymised retrospective analyses were covered by the German Hospital Law. We declare that all research was performed according to the code of conduct of the World Medical Association and the Declaration of Helsinki.

\section{Conflict of Interest Statement}

The authors declare no conflicts of interest.

\section{Funding Sources}

This study did not receive any specific grant from funding agencies in the public, commercial, or not-for-profit sector.

\section{Author Contributions}

The study was designed and conceived by S.M., K.S.R., and T.F. A.M.B. collected the data. Acquisition, analysis or interpretation of data was performed by S.M., K.S.R., A.M.B., and F.D. The manuscript was drafted by S.M., K.S.R., F.D., and P.R. Statistical analyses were carried out by S.M., K.S.R., and A.M.B. The manuscript was critically revised by all authors.

\section{References}

1 Ellis IO. Best Practice No 179: Guidelines for breast needle core biopsy handling and reporting in breast screening assessment. J Clin Pathol. 2004 Sep;57(9):897-902.

2 Sloane JP, Amendoeira I, Apostolikas N, Bellocq JP, Bianchi S, Boecker W, et al. Consistency achieved by 23 European pathologists from 12 countries in diagnosing breast disease and reporting prognostic features of carcinomas. European Commission Working Group on Breast Screening Pathology. Virchows Arch. 1999 Jan;434(1):3-10.

3 Forester ND, Lowes S, Mitchell E, Twiddy M. High risk (B3) breast lesions: What is the in- cidence of malignancy for individual lesion subtypes? A systematic review and meta-analysis. Eur J Surg Oncol. 2019 Apr;45(4):51927.

4 Houssami N, Ciatto S, Bilous M, Vezzosi V, Bianchi S. Borderline breast core needle histology: predictive values for malignancy in lesions of uncertain malignant potential (B3). Br J Cancer. 2007 Apr;96(8):1253-7.

5 Lee AH, Denley HE, Pinder SE, Ellis IO, Elston CW, Vujovic P, et al. Excision biopsy findings of patients with breast needle core biopsies reported as suspicious of malignancy (B4) or lesion of uncertain malignant poten- tial (B3). Histopathology. 2003 Apr;42(4): 331-6.

6 Bianchi S, Caini S, Renne G, Cassano E, Ambrogetti D, Cattani MG, et al. Positive predictive value for malignancy on surgical excision of breast lesions of uncertain malignant potential (B3) diagnosed by stereotactic vacuum-assisted needle core biopsy (VANCB): A large multi-institutional study in Italy. Breast. 2011 Jun;20(3):264-70.

7 Jacobs TW, Connolly JL, Schnitt SJ. Nonmalignant Lesions in Breast Core Needle Biopsies: To Excise or Not to Excise? Am J Surg Pathol. 2002 Sep;26(9):1095-110. 
8 Carder PJ, Khan T, Burrows P, Sharma N. Large volume "mammotome" biopsy may reduce the need for diagnostic surgery in papillary lesions of the breast. J Clin Pathol. 2008 Aug;61(8):928-33.

9 Kim MJ, Kim EK, Lee JY, Youk JH, Park BW, Kim SI, et al. Breast lesions with imaging-histologic discordance during US-guided 14G automated core biopsy: can the directional vacuum-assisted removal replace the surgical excision? Initial findings. Eur Radiol. 2007 Sep;17(9):2376-83.

10 Rageth CJ, O'Flynn EAM, Pinker K, KubikHuch RA, Mundinger A, Decker T, et al. Second International Consensus Conference on lesions of uncertain malignant potential in the breast (B3 lesions). Breast Cancer Res Treat. 2019 Apr;174(2):279-96.

11 Weigel S, Decker T, Korsching E, Biesheuve C, Wöstmann A, Böcker W, et al. Minimal Invasive Biopsy Results of "Uncertain Malignant Potential" in Digital Mammography Screening: High Prevalence but also High Predictive Value for Malignancy. RoFo. 2011 Aug;183(08):743-8.

12 Wen X, Cheng W. Nonmalignant Breast Papillary Lesions at Core-Needle Biopsy: A Metaanalysis of Underestimation and Influencing Factors. Ann Surg Oncol. 2013 Jan;20(1):94101

13 Calhoun BC, Collins LC. Recommendations for excision following core needle biopsy of the breast: a contemporary evaluation of the literature. Histopathology. 2016 Jan;68(1): 138-51.

14 Rageth CJ, O'Flynn EA, Comstock C, Kurtz C, Kubik R, Madjar H, et al. First International Consensus Conference on lesions of uncertain malignant potential in the breast (B3 lesions). Breast Cancer Res Treat. 2016 Sep; 159(2):203-13.

15 Abdulcadir D, Nori J, Meattini I, Giannotti E, Boeri C, Vanzi E, et al. Phyllodes tumours of the breast diagnosed as B3 category on image- guided 14-gauge core biopsy: Analysis of 51 cases from a single institution and review of the literature. Eur J Surg Oncol. 2014 Jul; 40(7):859-64.

16 Ditsatham C, Chongruksut W. Phyllodes tumor of the breast: diagnosis, management and outcome during a 10-year experience. Cancer Manag Res. 2019 Aug;11:7805-11.

17 de Beça FF, Rasteiro C, Correia A, Costa S, Amendoeira I. Improved malignancy prediction by B3 breast lesions subclassification. Ann Diagn Pathol. 2013 Oct;17(5):434-6.

18 Richter-Ehrenstein C, Maak K, Röger S, Ehrenstein T. Lesions of "uncertain malignant potential" in the breast (B3) identified with mammography screening. BMC Cancer. 2018 Dec;18(1):829.

19 Calhoun BC, Sobel A, White RL, Gromet M, Flippo T, Sarantou T, et al. Management of flat epithelial atypia on breast core biopsy may be individualized based on correlation with imaging studies. Mod Pathol. 2015 May; 28(5):670-6

20 Yu CC, Ueng SH, Cheung YC, Shen SC, Kuo WL, Tsai HP, et al. Predictors of Underestimation of Malignancy after Image-Guided Core Needle Biopsy Diagnosis of Flat Epithelial Atypia or Atypical Ductal Hyperplasia. Breast J. 2015 May-Jun May;21(3):224-32.

21 Menon S, Porter GJ, Evans AJ, Ellis IO, Elston CW, Hodi Z, et al. The significance of lobular neoplasia on needle core biopsy of the breast. Virchows Arch. 2008 May;452(5):473-9.

22 Page DL, Kidd TE, Dupont WD, Simpson JF, Rogers LW. Lobular neoplasia of the breast: Higher risk for subsequent invasive cancer predicted by more extensive disease. Hum Pathol. 1991 Dec;22(12):1232-9.

23 Moskovszky L, Berger B, Fleischmann A, Friedrich T, Helmchen B, Körner M, et al. Inter-observer reproducibility of classical lobular neoplasia (B3 lesions) in preoperative breast biopsies: a study of the Swiss Working Group of breast and gynecopathologists. J
Cancer Res Clin Oncol. 2020 Jun;146(6): 1473-8

24 O’Malley FP. Lobular neoplasia: morphology, biological potential and management in core biopsies. Mod Pathol. 2010 May;23(Suppl 2): S14-25.

25 El-Sayed ME, Rakha EA, Reed J, Lee AH, Evans AJ, Ellis IO. Predictive value of needle core biopsy diagnoses of lesions of uncertain malignant potential (B3) in abnormalities detected by mammographic screening. Histopathology. 2008 Dec;53(6):650-7.

26 Nagi CS, O'Donnell JE, Tismenetsky M, Bleiweiss IJ, Jaffer SM. Lobular neoplasia on core needle biopsy does not require excision. Cancer. 2008 May;112(10):2152-8.

27 Zhao C, Desouki MM, Florea A, Mohammed K, Li X, Dabbs D. Pathologic Findings of Follow-up Surgical Excision for Lobular Neoplasia on Breast Core Biopsy Performed for Calcification. Am J Clin Pathol. 2012 Jul;138(1): 72-8.

28 Rakha EA, Lee AH, Jenkins JA, Murphy AE, Hamilton LJ, Ellis IO. Characterization and outcome of breast needle core biopsy diagnoses of lesions of uncertain malignant potential (B3) in abnormalities detected by mammographic screening. Int J Cancer. 2011 Sep; 129(6):1417-24.

29 Pinder SE, Ellis IO. The diagnosis and management of pre-invasive breast disease: Ductal carcinoma in situ (DCIS) and atypical ductal hyperplasia $(\mathrm{ADH})$ - current definitions and classification. Breast Cancer Res. 2003 Oct;5(5).

30 Mayer S, Kayser G, Rücker G, Bögner D, Hirschfeld M, Hug C, et al. Absence of epithelial atypia in B3-lesions of the breast is associated with decreased risk for malignancy. Breast. 2017 Feb;31:144-9.

31 Leitlinienprogramm Onkologie: S3-Leitlinie Früherkennung, Diagnose, Therapie und Nachsorge des Mammakarzinoms. Version 4.3, 2020; AWMF Reg. Nr 032-045OL 